BULL. AUSTRAL. MATH. SOC.

VOL. 29 (1984), 243-248.

\title{
COMPARAISON RESULTATS POUR LA STABILITÉ PRATIQUE
}

\section{THOMAS KIVENTIDIS}

We develop some comparison theorems that connect the solutions of a differential system and its perturbation in a manner useful in the practical stability.

\section{Introduction-Définitions}

Considerons le système

$$
\dot{x}=f(t, x), x\left(t_{0}\right)=x_{0}, t_{0} \geqq 0, x_{0} \in \mathbb{R}^{n}
$$

et son perturbation système

$$
\dot{y}=f(t, y)+F(t, y), \quad y\left(t_{0}\right)=x_{0}
$$

où $f, u \in C\left(R^{+} \times D, R^{n}\right)$ et $D$ est une région convexe de $\mathbb{R}^{n}$.

On utilesera le leme qui suit (2):

LEMME. On suppose que le systeme (1) admet une solution unique $x\left(t, t_{0}, x_{0}\right)$. On assume, aussi, que $\phi\left(t, t_{0}, x_{0}\right) \equiv \frac{\partial}{\partial x_{0}} x\left(t, t_{0}, x_{0}\right)$ existe et il est continue pour $t \geqq t_{0}$ et que $\phi^{-1}\left(t, t_{0}, x_{0}\right)$ existe pour $t \geqq t_{0}$. Si $u(t)$ est une solution du systeme

$$
\dot{u}(t)=\phi^{-1}\left(t, t_{0}, x_{0}\right) F\left(t, x\left(t, t_{0}, x_{0}\right)\right), \quad u\left(t_{0}\right)=x_{0}
$$

alors une solution $y\left(t, t_{0}, x_{0}\right) d u(2)$ satisfait la relation

Received 25 October 1983.

Copyright Clearance Centre, Inc. Serial-fee code: 0004-9727/84 $\$ A 2.00+0.00$ 


$$
y\left(t, t_{o}, x\right)=x\left(t, t_{o}, u(t)\right)
$$

dans l'intervalle d'existence de la solution $u(t)$.

DÉFINITIONS (3). 1 . Le système (2) est pratiquement stable par rapport aux données $\left(\varepsilon_{1}, \varepsilon_{2}, \delta, t_{0},\|\|.\right), \varepsilon_{1} \leq \varepsilon_{2}$, si pour chaque solution $y(t)$, les conditions $\left\|y\left(t_{0}\right)\right\|<\varepsilon_{1}$ et $\|F(t, y)\| \leq \delta, \forall t \geq t_{0}$, $y \in \bar{B}\left(\varepsilon_{2}\right)=\left\{x \in \mathbb{R}^{n}:\|x\| \leq \varepsilon_{2}\right\}$, entrainent la relation $\|x(t)\|<\varepsilon_{2}, \forall t \geq t_{0}$.

2. Le système (2) est pratiquement asymptotiquement stable par rapport aux données $\left(\varepsilon_{1}, \varepsilon_{2}, \varepsilon_{3}, \delta, t_{0},\|\|.\right), \varepsilon_{2}<\varepsilon_{1} \leq \varepsilon_{3}$, si pour chaque solution $y(t)$ les conditions $\left\|y\left(t_{0}\right)\right\|<\varepsilon_{1}$, et $\|F(t, y)\| \leq \delta, \forall t \geq t_{o}, y \in \bar{B}\left(\varepsilon_{3}\right)$, entrainent: (i) la stabilitê pratique par rapport $\left(\varepsilon_{1}, \varepsilon_{3}, \delta, t_{0},\|\cdot\|\right)$, (ii) il existe $T>t_{0}$ tel que $\|y(t)\|<\varepsilon_{2}, \forall t \geq T$.

Si les définitions précédentes sont valables pour chaque $t_{1} \in\left(t_{0}, \infty\right)$, au place de $t_{0}$ nous avons les stabilités pratiques uniformes. On ajoute, encore, que pour $\delta=0$, nous avons les stabilités pratiques pour le système (1).

\section{Comparaison Théorèmes}

THÉORÈME 1. On assume que les conditions du Zemme (\$1) et Z'estimation

$$
\left\|\phi^{-1}\left(t, t_{0}, x_{0}\right)\right\| \leq \delta^{-1} g\left(t,\left\|x_{0}\right\|\right)
$$

se satisfont, où $g \in C\left(R^{+} \times R^{+}, R^{+}\right)$et le système

$$
\dot{u}=g(t, u), u\left(t_{0}\right)=u_{0} \geq 0
$$

est (uniformement) pratiquement stable par rapport aux données $\left(\alpha_{1}, \alpha_{2}, t_{0},||.\right), \alpha_{1} \leq \alpha_{2}$. En outre, on suppose que le systeme (1) est (uniformement) pratiquement stable par rapport aux donnés $\left(\alpha_{2}, \varepsilon_{2}, t_{0},\|\|.\right)$, $\alpha_{2} \leq \varepsilon_{2}$. Alors, le système (2) est (uniformement) pratiquement stable par rapport aux données $\left(\alpha_{2}, \varepsilon_{2}, \delta, t_{0},\|\|.\right), \alpha_{1} \leq \varepsilon_{2}$. 
DÉMONSTRATION. Par le lemme (\$1) nous avons la relation

$$
y\left(t, t_{0}, x_{0}\right)=x\left(t, t_{0}, U(t)\right)
$$

ou $y\left(t, t_{0}, x_{0}\right)$ est une solution du (2) et $U(t)$ est une solution du (3). si on a $\|F(t, x)\| \leq \delta, \forall t \geq t_{0},\|y\| \leq \varepsilon_{2}$, alors

$$
\left\|\phi^{-1}\left(t, t_{0}, x_{0}\right) F\left(t, t_{0}, x_{0}\right)\right\| \leq\left\|\phi^{-1}\left(t, t_{0}, x_{0}\right)\right\| \delta
$$

on met $m(t)=\|U(t)\|$, alors l'inégalité

$$
D^{+} m(t) \leq g(t, m(t))
$$

donne 1'estimation [1, Theorem 1.4.1]

$$
\|U(t)\|=m(t) \leq u\left(t, t_{o},\left\|x_{0}\right\|\right), t \geq t_{0}
$$

où $u\left(t, t_{0}, u_{0}\right)$ est la solution maximale du (4).

Par 1'hypothèse, si on a $\left\|x_{0}\right\|<\alpha_{2}$, alors

$$
\left\|x\left(t, t_{0}, x_{0}\right)\right\|<\varepsilon_{2}, \forall t \geq t_{0}
$$

et si $u_{0}<\alpha_{1}$ entraine $\|U(t)\| \leq\left|u\left(t, t_{0}, U(t)\right)\right|<\alpha_{2}$.

Mais par le lemme résulte que

$$
\left\|y\left(t, t_{0}, x_{0}\right)\right\|=\left\|x\left(t, t_{o}, U(t)\right)\right\|,
$$

et par conséquent, si $\left\|x_{0}\right\|<\varepsilon_{1}$, on a $\left\|y\left(t, t_{0}, x_{0}\right)\right\|<\varepsilon_{2}, \forall t \geq t_{0}$.

Quand nous avons la stabilité uniforme les relations ci-dessus sont valables pour l'initial temps $t_{1} \geq t_{0}$ (pour tous $t_{1} \geq t_{0}$ ).

THÉORÈME 2. On assume que les hypothèses du theoreme 1 se satisfont et que le systeme (4) est (uniformement) pratiquement stable par rapport aux donneses $\left(\alpha_{1}, \alpha_{2}, t_{0}, 1 . \mid\right), \alpha_{1} \leq \alpha_{2}$. En outre, on suppose que le systeme (1) est (uniformement) asymptotiquement pratiquement stable par rapport aux donnees $\left(\alpha_{1}, \varepsilon_{3}, \varepsilon_{2}, t_{0},\|\|.\right), \varepsilon_{3}<\alpha_{2} \leq \varepsilon_{2}$, alors le systeme (2) est 
(uniformément) asymptotiquement pratiquement stable par rapport aux donnees $\left(\alpha_{1}, \varepsilon_{3}, \varepsilon_{2}, \delta, t_{0},\|\|.\right), \varepsilon_{3}<\alpha_{1} \leq \varepsilon_{2}$.

DÉMONSTRATION. La (uniforme) stabilité pratique du système (2) par rapport aux données $\left(\alpha_{1}, \varepsilon_{2}, \delta, t_{0},\|\|.\right), \alpha_{1} \leq \varepsilon_{2}$, résulte par le théorème 1 .

Par l'hypothèse pour le système (1) il y a temps $T\left(\varepsilon_{3}, t_{0}\right)$ (resp.T $\left(\varepsilon_{3}\right)$ quand nous avons la stabilité uniforme) tel que

$$
\left\|x\left(t, t_{0}, x_{0}\right)\right\|<\varepsilon_{3}, \quad \forall t \geq T
$$

quand $\left\|x_{0}\right\|<\alpha_{2}$.

Mais, on a aussi $\|U(t)\| \leq\left|u\left(t, t_{0}, u_{0}\right)\right|<\alpha_{2}$, si $\left|u_{0}\right|<\alpha_{1}$.

Par conséquent, nous aurons

$$
\left\|y\left(t, t_{o}, x_{o}\right)\right\|=\left\|x\left(t, t_{o}, U(t)\right)\right\|<\varepsilon_{3}, \forall t \geq T
$$

quand $\left\|x_{0}\right\|<\alpha_{1}$.

\section{Exemple}

On considère le système $\dot{x}=A(t) x, x\left(t_{0}\right)=x_{0},(1)$, alors on a $\phi\left(t, t_{0}, x_{0}\right)=\phi\left(t, t_{0}\right)$, où $\phi\left(t, t_{0}\right)$ est la résolvante du (1).

Si on a

$$
\left\|\phi^{-1}\left(t, t_{0}\right)\right\| \leq\left\|\delta^{-1} \lambda(t)\right\|,
$$

où $\lambda(t)$ est telle que

$$
0 \leq \int_{t_{0}}^{\infty} \lambda(t) d t=\varepsilon<\infty, \lambda(t) \geq 0,
$$

alors, on prend $g\left(t,\left\|x_{0}\right\|\right)=\lambda(t)$.

Le système (4), en ce cas, est $\dot{u}=\lambda(t)$ et il a les solutions

$$
u(t)=\int_{t_{1}}^{t} \lambda(s) d s+u_{0}, u\left(t_{1}\right)=u_{0} \geq 0, t_{1} \geq t_{0} .
$$

Par conséquent, le système (4) est uniformément pratiquement stable 
par rapport aux données $\left(\alpha_{1}, \alpha_{2}, t_{1},||.\right), \alpha_{1} \leq \alpha_{2}, t_{1} \geq t_{0}$, où $u_{0}<\alpha_{1}$ et $\varepsilon+u_{0}<\alpha_{2}$.

Donc, si le système (1) est (uniformément) pratiquement stable (resp. (uniformément) asymptotiquement pratiquement stable) par rapport aux données $\left(\alpha_{2}, \varepsilon_{2}, t_{0},\|\|.\right), \alpha_{2} \leq \varepsilon_{2},\left(\operatorname{resp} .\left(\alpha_{2}, \varepsilon_{3}, \varepsilon_{2}, t_{o},\|\cdot\|\right)\right.$, $\varepsilon_{3}<\alpha_{2} \leq \varepsilon_{2}$ ), alors par le théorème 1 (resp. par le théorème 2) résulte que le système (2)

$$
\dot{y}=A(t) y+E(t, y), y\left(t_{0}\right)=x_{0}
$$

est (uniformément) pratiquement stable (resp. (uniformément) asymptotiquement pratiquement stable) par rapport aux données $\left(\alpha_{1}, \varepsilon_{2}, \delta, t_{0},\|\|.\right)$, $\alpha_{1} \leq \alpha_{2},\left(\operatorname{resp} .\left(\alpha_{1}, \varepsilon_{3}, \varepsilon_{2}, \delta, t_{0},\|\|.\right), \varepsilon_{3}<\alpha_{1} \leq \varepsilon_{2}\right)$.

En particulier, on considère le système $\dot{x}=A(t) x$, où

$$
A(t)=\left(\begin{array}{ll}
0 & 1 \\
-(1+q(t)) & 0
\end{array}\right)
$$

Evidemment la maximale characteristique valeur de la matrice $\frac{1}{2}\left(A+A^{T}\right)$ $\left(A^{T} \quad\right.$ transposée de $\left.A\right)$ est $\Lambda(t)=\frac{1}{2}|q(t)|$.

si on a

$$
\int_{t_{1}}^{t_{2}} \Lambda(s) d s<\log \frac{\varepsilon_{2}}{\alpha_{2}}, \forall t_{2}>t_{1} \geq t_{0}, \alpha_{2}<\varepsilon_{2},
$$

alors [3, 53, p.31] le système (1) est uniformément pratiquement stable par rapport aux données $\left(\alpha_{2}, \varepsilon_{2}, t_{0},\|\|.\right), \alpha_{2}<\varepsilon_{2}$.

En outre, si les relations ci-dessus se verifient pour $\phi^{-1}\left(t, t_{0}\right)$ et $g(t, u)$, on peut utiliser les théorèmes 1 et 2 pour prendre les résultats pour la stabilité pratique du système (2). 


\section{Références}

[1] V. Lakshmikantham and S. Leela, Differential and Integral Inequalities Vol. 1 (Academic Press, New York, 1969).

[2] M. Lord and A.R. Mitchell, "A new approach to the method of nonlinear variation of constants", Appl. Math. Comput. 4 (1978), 95-105.

[3] T. Kiventidis, Practical stability under perturbations, (Ph.D Thesis, Thessaloniki, 1978).

Département de Mathématique,

Universite de Thessaloniki,

Thessaloniki,

Grèce. 